\title{
Examination of Minimum Time Step, from Modified Heisenberg Uncertainty Principle, Inflaton Physics and Black Hole Physics
}

\author{
Andrew Walcott Beckwith \\ Physics Department, College of Physics, Chongqing University Huxi Campus, Chongqing, China \\ Email: Rwill9955b@gmail.com, abeckwith@uh.edu
}

How to cite this paper: Beckwith, A.W. (2017) Examination of Minimum Time Step, from Modified Heisenberg Uncertainty Principle, Inflaton Physics and Black Hole Physics. Journal of High Energy Physics, Gravitation and Cosmology, 3, 3945.

http://dx.doi.org/10.4236/jhepgc.2017.31007

Received: September 5, 2016

Accepted: November 21, 2016

Published: November 25, 2016

Copyright $\odot 2017$ by author and Scientific Research Publishing Inc. This work is licensed under the Creative Commons Attribution International License (CC BY 4.0).

http://creativecommons.org/licenses/by/4.0/

\begin{abstract}
First, we calculate the minimum length for the creation of a $10^{45} \mathrm{~Hz}$ relic Gravitational wave. Next, we look Padamababhan's inflaton physics, and work done by the author for a modified Heisenberg Uncertainty principle for constraints on a minimum time step. Sciama's work in "Black hole explosions" (1982) gives us a linkage between a decay rate for black holes, in terms of a life time, and the mass, $M$ of the black hole, which when combined with a simple exposition from Susskind and Hrabovsky (2013) for the most basic evolution the time change in energy $E(t)$, which is how we form a first order treatment of the square of a minimum time step $\Delta t$. We then reference what was done by $\mathrm{Ng}$ (2008) as far as infinite quantum statistics, for entropy as a particle count, and from first principle get constraints upon entropy production, as a function of boundaries on minimum time step. We assume massive Gravity, and obtain a peak $10^{36}$ Giga Hertz frequency range $\left(10^{45} \mathrm{Hertz}\right)$ for relic Gravitational waves, and Gravitons.
\end{abstract}

\section{Keywords}

Massive Gravity, Inflaton Physics, Infinite Quantum Statistics,

Modified Poisson's Equation

\section{Introduction}

First of all we calculate a minimum length, $r$, and this on the basis of a Poissons equation given in [1], and then afterwards we will secondly get to calculation of the time step. This is to obtain a Pre inflationary value of the initial time step, $\Delta t$, and then from there we will use an order of magnitude estimate as to the initial mass $M$ of a black hole, to lead to our final conditions. 


\section{Modified Poissons Equation [1] Applied to Our Problem}

We will first of all refer to two necessary and sufficient conditions for the onset of a massive graviton given in [1], and combined with Padmanablan's reference [2]. This heavily borrows from [3]. i.e. what we will be doing is to re do the reference calculations given in [1] with

$$
\left(\nabla^{2}+\left[\lambda^{-2}=\left(\frac{m_{\text {graviton }} c}{\hbar}\right)^{2}\right]\right)\left[U=\frac{G m}{r} \cdot \exp \left[(-r / \lambda)=\left(\frac{r \cdot m_{\text {graviton }} c}{\hbar}\right)\right]\right]=-4 \pi G \rho
$$

Here, we will be using in the Pre-Planckian potential the inputs from the data usually associated with [2]

$$
\begin{aligned}
& a \approx a_{\min } t^{\gamma} \\
& \Leftrightarrow \phi \approx \sqrt{\frac{\gamma}{4 \pi G}} \cdot \ln \left\{\sqrt{\frac{8 \pi G V_{0}}{\gamma \cdot(3 \gamma-1)}} \cdot t\right\} \\
& \Leftrightarrow V \approx V_{0} \cdot \exp \left\{-\sqrt{\frac{16 \pi G}{\gamma}} \cdot \phi(t)\right\}
\end{aligned}
$$

In other words, we will be using the inflation given by

$$
\phi \approx \sqrt{\frac{\gamma}{4 \pi G}} \cdot \ln \left\{\sqrt{\frac{8 \pi G V_{0}}{\gamma \cdot(3 \gamma-1)}} \cdot t\right\}
$$

If so, then our approximation is to call the Potential in Equation (2) to be the same as $\mathrm{U}$ in Equation (1), and then with re arrangements we come up with the following

$$
\left[\frac{\mathrm{d}^{2}}{\mathrm{~d} r^{2}}+\left(\frac{m_{\text {graviton }} c}{\hbar}\right)^{2}\right] \cdot\left(\frac{r^{-1} \gamma \cdot(3 \gamma-1)}{32 \pi^{2}}\right)=G \cdot \rho
$$

Then, after algebra, we have the following

$$
\left(m_{\text {graviton }}\right)^{2} \approx\left[\frac{32 \pi^{2} r \cdot \hbar^{2} \cdot G \cdot \rho}{c^{2} \cdot \gamma \cdot(3 \gamma-1)}-\frac{16 \pi^{2} r^{-1} \cdot \hbar^{2} \cdot G}{c^{2} \cdot \gamma \cdot(3 \gamma-1)}\right]
$$

The quadratic Equation this engenders is, how to say

$$
\begin{aligned}
& \left(m_{\text {graviton }}\right)^{2} \approx\left[\frac{32 \pi^{2} r \cdot \hbar^{2} \cdot G \cdot \rho}{c^{2} \cdot \gamma \cdot(3 \gamma-1)}-\frac{16 \pi^{2} r^{-1} \cdot \hbar^{2} \cdot G}{c^{2} \cdot \gamma \cdot(3 \gamma-1)}\right] \\
& \Rightarrow r^{2}-\frac{r \cdot\left(m_{\text {graviton }}\right)^{2}}{\left(\frac{32 \pi^{2} \hbar^{2} \cdot G \cdot \rho}{c^{2} \cdot \gamma \cdot(3 \gamma-1)}\right)}-\frac{\left(\frac{16 \pi^{2} \cdot \hbar^{2} \cdot G}{c^{2} \cdot \gamma \cdot(3 \alpha-1)}\right)}{\left(\frac{32 \pi^{2} \hbar^{2} \cdot G \cdot \rho}{c^{2} \cdot \gamma(3 \gamma-1)}\right)} \doteq r^{2}-\frac{r \cdot\left(m_{\text {graviton }}\right)^{2}}{\left(\frac{32 \pi^{2} \hbar^{2} \cdot G \cdot \rho}{c^{2} \cdot \gamma \cdot(3 \gamma-1)}\right)}-\frac{1}{2 \rho}=0
\end{aligned}
$$

We will, in the latter part of this document, associate

$$
\rho \sim \Delta E / r^{3}
$$

And after defining as such work with Equation (7) put into Equation (6) we obtain

$$
\begin{aligned}
& r^{2}-\frac{r^{4} \cdot\left(m_{\text {graviton }}\right)^{2}}{\left(\frac{32 \pi^{2} \hbar^{2} \cdot G}{c^{2} \cdot \gamma \cdot(3 \gamma-1)}\right) \Delta E}-\frac{r^{3}}{2 \Delta E}=0 \\
\Rightarrow & r^{2}+r \cdot\left[\left(\frac{32 \pi^{2} \hbar^{2} \cdot G}{c^{2} \cdot \gamma \cdot(3 \gamma-1)}\right) \frac{1}{2 \cdot\left(m_{\text {graviton }}\right)^{2}}\right]-\left(\frac{32 \pi^{2} \hbar^{2} \cdot G}{c^{2} \cdot \gamma \cdot(3 \gamma-1)}\right) \frac{\Delta E}{\left(m_{\text {graviton }}\right)^{2}}=0
\end{aligned}
$$


We will from here, insert, in the value of $\Delta E$ from the HUP given in [3] and also compare it to the work given by Susskind and Hrabovsky in [4] and from there get a linkage of a radial distance, inflaton physics, and a time step $\Delta t$.

\section{HUP, as Used in This Problem}

Begin with [3]

$$
\Delta E \sim \frac{\hbar}{\delta t \cdot a_{\min }^{2} \phi_{\text {inf }}}
$$

In [3] we make the assumption, namely $\phi \approx \sqrt{\frac{\gamma}{4 \pi G}} \cdot \ln \left\{\sqrt{\frac{8 \pi G V_{0}}{\gamma \cdot(3 \gamma-1)}} \cdot t\right\}$, then Equation (9) becomes

$$
\Delta E \sim \frac{\hbar}{\delta t \cdot a_{\min }^{2} \sqrt{\frac{\gamma}{4 \pi G}} \cdot \ln \left\{\sqrt{\frac{8 \pi G V_{0}}{\gamma \cdot(3 \gamma-1)}} \cdot \delta t\right\}}
$$

Then

$$
\begin{aligned}
& r^{2}+r \cdot\left[\left(\frac{32 \pi^{2} \hbar^{2} \cdot G}{c^{2} \cdot \gamma \cdot(3 \gamma-1)}\right) \frac{1}{2 \cdot\left(m_{\text {graviton }}\right)^{2}}\right] \\
& +\left(\frac{32 \pi^{2} \hbar^{2} \cdot G}{c^{2} \cdot \gamma \cdot(3 \gamma-1)}\right) \cdot \frac{\hbar}{\left(m_{\text {graviton }}\right)^{2} \delta t \cdot a_{\min }^{2} \sqrt{\frac{\gamma}{4 \pi G}} \cdot\left|\ln \left\{\sqrt{\frac{8 \pi G V_{0}}{\gamma \cdot(3 \gamma-1)}} \cdot \delta t\right\}\right|}=0
\end{aligned}
$$

This will be further elaborated upon in this document.

\section{Examining the Consequences of Equation (10) and Equation (11)}

Using the CRC tables, we obtain

$$
\begin{aligned}
& r=\frac{-1}{2} \cdot\left(\frac{32 \pi^{2} \hbar^{2} \cdot G}{c^{2} \cdot \gamma \cdot(3 \gamma-1)}\right) \frac{1}{2 \cdot\left(m_{\text {graviton }}\right)^{2}} \\
& \cdot\left[1-\sqrt{1-16 \frac{\hbar \cdot\left(m_{\text {gravion }}\right)^{2}}{\delta t \cdot a_{\min }^{2} \sqrt{\frac{\gamma}{4 \pi G}} \cdot \ln \left\{\sqrt{\frac{8 \pi G V_{0}}{\gamma \cdot(3 \gamma-1)}} \cdot \delta t\right\}\left[\left(\frac{32 \pi^{2} \hbar^{2} \cdot G}{c^{2} \cdot \gamma \cdot(3 \gamma-1)}\right)\right]^{2}}}\right]
\end{aligned}
$$

Now, then, we can form a force equation, and do it via

$$
F(\text { force-graviton })=-\nabla V(\text { potential })
$$

The expression $V$ (potential) is formed from the inflaton, and we get From Padmanablah, using [1] if $a(t)=a_{0} t^{\gamma}$, then by [1]

$$
V(t)=V_{0}\left(\frac{\gamma(3 \gamma-1)}{8 \pi G V_{0}}\right)^{1 / \sqrt{2}} \frac{c^{\sqrt{2}}}{t^{\sqrt{2}}}=V_{0}\left(\frac{\gamma(3 \gamma-1)}{8 \pi G V_{0}}\right)^{1 / \sqrt{2}} \frac{c^{2}}{r^{\sqrt{2}}}
$$


Then, by Equation (13)

$$
F(\text { force-graviton })=-\nabla V(\text { potential })=\sqrt{2} V_{0}\left(\frac{\gamma(3 \gamma-1)}{8 \pi G V_{0}}\right)^{1 / \sqrt{2}} \frac{c^{2}}{r^{1+\sqrt{2}}}
$$

Now, the time derivative of energy, is [4]

$$
\frac{\mathrm{d} E}{\mathrm{~d} t}=v(\text { velocity }) \cdot(m(\text { mass }) \cdot a(\text { acceleration })-F(\text { force }))
$$

Then, if the derivatives $\mathrm{d} E / \mathrm{d} t$ become instead incremental

$$
\Delta E \Delta t=c(\Delta t)^{2} \cdot\left(m(\text { mass }) \cdot a(\text { acceleration })-\sqrt{2} V_{0}\left(\frac{\gamma(3 \gamma-1)}{8 \pi G V_{0}}\right)^{1 / \sqrt{2}} \frac{c^{2}}{r^{1+\sqrt{2}}}\right)
$$

Then, using $\Delta E \sim \frac{\hbar}{\delta t \cdot a_{\text {min }}^{2} \phi_{\text {inf }}}$

$$
\begin{aligned}
& a_{\min }^{2} \sqrt{\frac{\gamma}{4 \pi G}} \cdot \ln \left\{\sqrt{\frac{8 \pi G V_{0}}{\gamma \cdot(3 \gamma-1)}} \cdot \delta t\right\} \\
& =c(\Delta t)^{2} \cdot\left(m(\text { mass }) \cdot a(\text { acceleration })-\sqrt{2} V_{0}\left(\frac{\gamma(3 \gamma-1)}{8 \pi G V_{0}}\right)^{1 / \sqrt{2}} \frac{c^{2}}{r^{1+\sqrt{2}}}\right)
\end{aligned}
$$

Now, set $\delta t=(\Delta t)$, and assume in the Pre-Planckian regime, that $m($ mass $) \cdot a($ acceleration $) \sim 0$

$$
\frac{\hbar}{(\Delta t) \cdot a_{\min }^{2} \sqrt{\frac{\gamma}{4 \pi G}} \cdot \ln \left\{\sqrt{\frac{8 \pi G V_{0}}{\gamma \cdot(3 \gamma-1)}} \cdot(\Delta t)\right\}}=c(\Delta t) \cdot\left(-\sqrt{2} V_{0}\left(\frac{\gamma(3 \gamma-1)}{8 \pi G V_{0}}\right)^{1 / \sqrt{2}} \frac{c^{2}}{r^{1+\sqrt{2}}}\right)
$$

Then Equation (12) becomes

$$
\begin{aligned}
r= & \frac{-1}{2} \cdot\left(\frac{32 \pi^{2} \hbar^{2} \cdot G}{c^{2} \cdot \alpha \cdot(3 \alpha-1)}\right) \frac{1}{2 \cdot\left(m_{\text {graviton }}\right)^{2}} \\
& \left.\cdot 1-\sqrt{1+16 c \cdot(\Delta t) \cdot\left(\sqrt{2} V_{0}\left(\frac{\gamma(3 \gamma-1)}{8 \pi G V_{0}}\right)^{1 / \sqrt{2}} \frac{\left(c \cdot m_{\text {graviton }}\right)^{2}}{r^{1+\sqrt{2}}}\right)^{2}}\right]
\end{aligned}
$$

We then get a positive $\mathrm{r}$ value, and up to a point this is equal to Planck Length $\sim 10^{-33}$ centimeters

To covert this to time, remove the spatial component on the right hand side of Equation (20) and this is done via

$$
\begin{aligned}
r= & \frac{-1}{2} \cdot\left(\frac{32 \pi^{2} \hbar^{2} \cdot G}{c^{2} \cdot \alpha \cdot(3 \alpha-1)}\right) \frac{1}{2 \cdot\left(m_{\text {graviton }}\right)^{2}} \\
& \cdot\left[1-\sqrt{1+16 c \cdot(\Delta t) \cdot\left(\sqrt{2} V_{0}\left(\frac{\gamma(3 \gamma-1)}{8 \pi G V_{0}}\right)^{1 / \sqrt{2}} \frac{\left(c \cdot m_{\text {graviton }}\right)^{2} c^{1+\sqrt{2}}}{(\Delta t)^{1+\sqrt{2}}}\right)^{2}}\right]
\end{aligned}
$$


We then get a positive $r$ value, and up to a point this is equal to Planck Length $\sim 10^{-33}$ centimeters

This, above, puts an incredibly tight set of constraints upon the minimum time step. It also assume, that we are looking at a rest mass for the graviton of the order of $10^{-62}$ grams. The above Equation (21) is arguing for a peak $10^{36}$ Giga Hertz frequency range $\left(10^{45}\right.$ Hertz) for relic Gravitational waves, and Gravitons.

\section{What Can Be Said Now about the Sciama Black Hole Explosion Idea? (1982)}

Sciama [5] in 1982 argued for the lifetime of a black hole, of mass $M$, that the following holds

$t($ black-hole-lifetime $) \sim 10^{10} \cdot\left(M / 10^{15} \text { grams }\right)^{3}$ years $\sim 3.154 \times 10^{17} \cdot\left(M / 10^{15} \text { grams }\right)^{3}$ sec

Here, if the time is about $10^{-44}$ seconds (Planck time), then $M \sim 10^{-7}$ grams. If so, then, according to [6], Calmert, et al. about $0.1 \%$ of the energy emitted, in the traditional 4 dimensional black hole $(3+1$ dimensions) would be gravitons

Then, $M \sim 10^{-7}$ grams becomes linked to Gravitons according to

$$
\left.M \text { ( } \text { primoridial-B.H.) } \sim 10^{-7} \text { grams } \Rightarrow M \text { (gravitons }\right) \sim 10^{-10} \text { grams }
$$

This would mean then 1 primordial black hole would produce, if the mass of a graviton is $10^{-62}$ grams [7]

$$
\text { total }-M \text { (gravitons) } \sim 10^{-10} \text { grams } \Rightarrow 10^{52} \text { gravitons }
$$

Assuming there would be say $10^{3}$ initial primordial black holes, we would then be obtaining $10^{55}$ relic gravitons.

Note that if the size of the presumed $\Delta t$ expanded, this would lead to a commensurate increase in relic gravitons.

\section{Conclusions}

We will, as a start, incorporate Ng's infinite quantum statistics idea, of entropy being equivalent to a count of particles, i.e. by [8]

$$
S \text { (entropy) \# gravitons }
$$

For relic conditions this implies about $10^{55}$ for relic entropy, as opposed to $10^{120}$ today for the existing entropy and this also implies an enormously high initial graviton initial frequency a peak $10^{36}$ Giga Hertz frequency range $\left(10^{45}\right.$ Hertz) for relic Gravitational waves.

Relic graviton frequency, if produced in the beginning of inflation would be by necessity red shifted down, by inflation. i.e. conceivably, if the $10^{45} \mathrm{~Hz}$ was de facto, it may mean present $\mathrm{GW}$ of the value of $10^{5}$ or so, i.e. a 40 magnitude drop in frequency range. i.e. if the generation of GW were at the very start of inflation, it may mean up to a 60 magnitude drop, and a correspondingly enormously low GW frequency. However, the degree of the drop, i.e. say with a 40 magnitude (low end) to a 62 magnitude drop, would say much about a proof about the degree and magnitude of the rapidity of inflation [9]. Which would be of critical import as to cosmology. Having said that there are some serious physical issues to keep in mind while doing this analysis. 
Furthermore, we should keep in mind the physics incorporated in [10] [11] i.e. as to the work of LIGO. i.e. it is important to keep in mind that in addition, that [12] has confirmed that a subsequent analysis of the event GW150914 by the LSC constrained the graviton Compton wavelength of those alternative theories of gravity in which the graviton is massive and placed a level of $90 \backslash \%$ confidence on the lower bound of $10^{\wedge}\{13\}$ $\mathrm{km}$ for a Compton wavelength of the graviton. Doing these sort of vetting protocols in line with being consistent with investigation as to a real investigation as to the fundamental nature of gravity. i.e. is this a way to show if general relativity is the final theory of gravitation. i.e., if massive gravity is confirmed, as give $\mathrm{n}$ in [13] then GR is perhaps to be replaced by a scalar-tensor theory, as has been shown by Corda.

In addition, note that [14] references how, in terms of refinement of gravitational wave detectors, that Second generation gravitational wave detectors require high power lasers with several 100W of output power and with very low temporal and spatial fluctuations would be helpful in terms of resolution perhaps aiding in obtaining resolution of the graviton mass as given in [11], which is in turn also commensurate with [12], as far as precise resolution of massive gravity's foot print, which in turn will aid in using Corda's insights as given in [13].

Refinements of instrumentation may be doable if the nature of the graviton, and its primordial genesis are understood more thoroughly, i.e. as discussed in [14].

Finally, all this may tie into experimental vetting and confirmation of the work by Corda, as given in [15], i.e. the nature of the inflaton, as an observed experimental quantity. This in conjunction with refinements in instrumentation brought up in [16].

\section{Acknowledgements}

This work is supported in part by National Nature Science Foundation of China grant No. 11375279.

\section{References}

[1] Poisson, E. and Will, C. (2014) Gravity, Newtonian, Post Newtoninan, Relativistic. Cambridge University Press, Cambridge, UK. https://doi.org/10.1017/CBO9781139507486

[2] Padmanabhan, T. (2005) Understanding Our Universe, Current Status and Open Issues. In: Ashatekar, A., Ed., 100 Years of Relativity, Space-Time Structure: Einstein and Beyond, World Scientific Publishing Co. Pte. Ltd., Singapore, 175-204. http://arxiv.org/abs/gr-qc/0503107

[3] Beckwith, A. (2016) Gedanken Experiment for Refining the Unruh Metric Tensor Uncertainty Principle via Schwarzschild Geometry and Planckian Space-Time with Initial Nonzero Entropy and Applying the Riemannian-Penrose Inequality and Initial Kinetic Energy for a Lower Bound to Graviton Mass (Massive Gravity). Journal of High Energy Physics, Gravitation and Cosmology, 2, 106-124. https://doi.org/10.4236/jhepgc.2016.21012

[4] Susskind, L. and Hrabovsky, G. (2013) The Theoretical Minimum. Basic Books, New York.

[5] Sciama, D. (1982) Black Hole Explosions. In: Terzian, Y. and Bilson, E.M., Eds., Cosmology and Astrophysics. Essays in Honor of Thomas Gold, Cornell University Press, Ithaca, 83-98.

[6] Calmert, X., Carr, B. and Winstanley, E. (2014) Quantum Black Holes. Springer Briefs in Physics, Springer Verlag, Heidelberg. https://doi.org/10.1007/978-3-642-38939-9

[7] Goldhaber, A. and Nieto, M. (2010) Photon and Graviton Mass Limits. Reviews of Modern 
Physics, 82, 939-979. https://arxiv.org/abs/0809.1003 https://doi.org/10.1103/RevModPhys.82.939

[8] Ng, Y.J. (2008) Spacetime Foam: From Entropy and Holography to Infinite Statistics and Nonlocality. Entropy, 10, 441-461. https://doi.org/10.3390/e10040441

[9] Freese, K. (1992) Natural Inflation. In: Nath, P. and Recucroft, S., Eds., Particles, Strings, and Cosmology, Northeastern University, March 25-30, 1991, World Scientific Publishing company, Pte. Ltd., Singapore, 408-428.

[10] Abbott, B., et al., LIGO Scientific Collaboration and Virgo Collaboration (2016) Observation of Gravitational Waves from a Binary Black Hole Merger. Physical Review Letters, 116, Article ID: 061102.

[11] Abbott, B., et al., LIGO Scientific Collaboration and Virgo Collaboration (2016) GW151226: Observation of Gravitational Waves from a 22-Solar-Mass Binary Black Hole Coalescence. Physical Review Letters, 116, Article ID: 241103.

[12] Abbott, B., et al. Tests of General Relativity with GW150914. https://arxiv.org/pdf/1602.03841.pdf

[13] Corda, C. (2009) Interferometric Detection of Gravitational Waves: The Definitive Test for General Relativity. International Journal of Modern Physics D, 18, 2275-2282. https://arxiv.org/abs/0905.2502 https://doi.org/10.1142/S0218271809015904

[14] Willke, B., et al. Stabilized High Power Laser for Advanced Gravitational Wave Detectors. http://iopscience.iop.org/article/10.1088/1742-6596/32/1/040/meta;jsessionid=B072B9D72E 7EB4F993D70061EEE365CF.c5.iopscience.cld.iop.org

[15] Corda, C. (2012) Gravity's Primordial Breath. Electronic Journal of Theoretical Physics, EJTP, 9, 1-10. http://www.ejtp.com/articles/ejtpv9i26p1.pdf

[16] Flaminico, R., et al. (2010) A Study of Coating Mechanical and Optical Losses in View of Reducing Mirror Thermal Noise in Gravitational Wave Detectors. Classical and Quantum Gravity, 27, Article ID: 084030 https://doi.org/10.1088/0264-9381/27/8/084030

Submit or recommend next manuscript to SCIRP and we will provide best service for you:

Accepting pre-submission inquiries through Email, Facebook, LinkedIn, Twitter, etc. A wide selection of journals (inclusive of 9 subjects, more than 200 journals)

Providing 24-hour high-quality service

User-friendly online submission system

Fair and swift peer-review system

Efficient typesetting and proofreading procedure

Display of the result of downloads and visits, as well as the number of cited articles

Maximum dissemination of your research work

Submit your manuscript at: http://papersubmission.scirp.org/

Or contact jhepgc@scirp.org 